\title{
HAARIGE DIAGNOSEN
}

\section{Alopecia areata: Kopfprobleme außen und innen}

- Alopecia areata geht nicht selten mit psychiatrischen Erkrankungen einher. Was dabei zuerst kommt, ist nicht immer ausgemacht.

Welche psychiatrischen Diagnosen bei Patienten mit Alopecia areata gestellt werden, haben taiwanesische Dermatologen anhand der nationalen Forschungsdatenbank der Krankenversicherungen eruiert. Analysiert wurden die Akten von 5117 Alopeziepatienten und 20468 Kontrollpersonen.

$8,1 \%$ der Alopecia-areata-Patienten und $6,1 \%$ der Kontrollen litten an einer psychiatrischen Erkrankung, ein signifikanter Unterschied $(p<0,01)$. Signifikant gehäuft traten bei Alopeziepatienten Angststö-

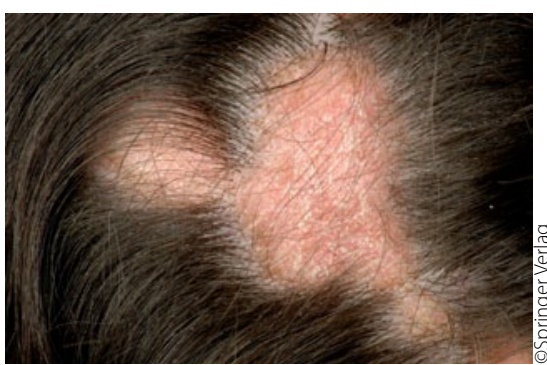

Zeichen einer kranken Psyche?

rungen (5,0\% der Patienten), Depressionen $(2,9 \%)$ und Zwangserkrankungen $(0,5 \%)$ auf. Fielen die Haare bereits vor dem 20. Lebensjahr aus, waren die Patienten häufig depressiv. Ängste wurden vor allem bei Patienten beobachtet, deren Alopecia areata im Alter zwischen 20 und 39 begonnen hatte. Zwangsstörungen traten besonders bei Erkrankung nach dem 40. Lebensjahr auf.

Interessanterweise gingen mehr als 50\% der psychiatrischen Leiden der Alopezie voraus. Die taiwanesischen Forscher sehen dies als Hinweis, die psychiatrischen Probleme nicht nur als Reaktion auf den Haarausfall zu deuten. Vielmehr könnten stressbedingte neuroendokrin-immunologische Mechanismen in die Pathogenese sowohl der Alopezie wie der psychiatrischen Erkrankungen eingreifen.

$\mathrm{RB}$ -

Chu S-Y et al. Br J Dermatol 2012; DOI: 0.1111/j.13652133.2011.10714.x

\section{AHA-Empfehlungen für "Safer Sex" bei kardiovaskulären Erkrankungen}

\section{Sex-Tipps für Herzkranke}

- Bevor Patienten mit neu festgestellter kardiovaskulärer Erkrankung wieder sexuell aktiv werden, sollte eine Medikamentenanamnese und körperliche Untersuchung stattfinden. Wenn sich dabei ein niedriges Risiko für kardiovaskuläre Komplikationen ergibt, spricht nichts gegen Sex.

- Wenn das kardiovaskuläre Risiko nicht niedrig oder nicht bekannt ist, wird ein Belastungstest empfohlen. Sexuelle Aktivität ist unproblematisch, wenn die Patienten dabei ohne Probleme mindestens 3-5 MET schaffen.

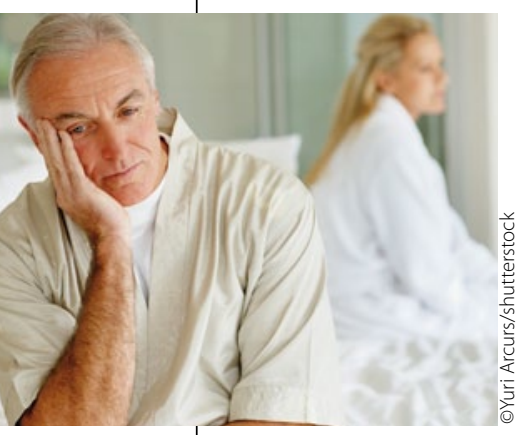

- Das Risiko für kardiovaskuläre Komplikationen beim Sex kann durch kardiale Rehabilitationsmaßnahmen und Sport gesenkt werden.

- Patienten mit instabiler, dekompensierter und/oder schwerer symptomatischer kardiovaskulärer Erkrankung sollten solange auf sexuelle Aktivität verzichten, bis sie stabilisiert und optimal behandelt sind.

- Bedenken wegen möglicher nachteiliger Auswirkungen auf die Sexualität dürfen kein Grund sein, auf die Verordnung wirksamer Herz-Kreislauf-Medikamente zu verzichten.

- Medikamente zur Behandlung der erektilen Dysfunktion, sprich PDE-5-Hemmer, werden bei stabiler kardiovaskulärer Erkrankung als sicher erachtet. PDE-5-Hemmer sind jedoch verboten, wenn die Patienten Nitrate einnehmen. Umgekehrt sollten Patienten, die einen PDE-5-Hemmer einnehmen, danach (je nach Substanz) 24 bis 48 Stunden lang kein Nitrat anwenden.

- Prämenopausale Frauen mit kardiovaskulären Erkrankungen sollten bezüglich der Sicherheit von Kontrazeptiva und Schwangerschaft beraten werden.

- Postmenopausale Frauen mit kardiovaskulären Erkrankungen sollten zur Behandlung einer Dyspareunie kein systemisches Östrogen erhalten.

- Nach einem kardialen Ereignis, bei einer neu diagnostizierten kardiovaskulären Erkrankung und nach der Implantation eines Defibrillators sollte im Rahmen der ärztlichen Beratung auch das Thema "Sex" angesprochen werden.

Circulation, online first, Jan 19, 2012

\section{ANTIDEPRESSIVA IM ALTER Auch SSRI erhöhen Sturzgefahr}

— Nicht nur anticholinerge Trizyklika, auch moderne SSRI erhöhen offenbar bei Demenzpatienten das Sturzrisiko. Vor allem die Kombination von SSRI mit Hypnotika und Sedativa sollten Ärzte meiden.

Forscher aus Rotterdam haben Daten von 248 Demenzpatienten eines Pflegeheims ausgewertet. Insgesamt dokumentierten sie 683 Stürze bei 152 Patienten. Etwa ein Drittel der Stürze führte zu Verletzungen. Die Patienten bekamen an über $16 \%$ aller Tage ein Antidepressivum, davon an 13\% aller Tage einen SSRI. In dieser Zeit war die Rate verletzungsträchtiger Stürze höher als ohne antidepressive Medikation, und zwar um den Faktor 3, wenn die Patienten die volle Tagesdosis eines SSRI erhielten, um 73\% bei der halben Tagesdosis und um über 30\% bei einem Viertel der Tagesdosis. Ein fast ähnlich hohes dosisabhängiges Sturzpotenzial ergab sich für Hypnotika und Sedativa. Am häufigsten stürzten die Patienten, wenn sie eine Kombination solcher Medikamente mit SSRI bekamen. Für diesen Fall errrechnete sich ein über siebenfach erhöhtes Sturzrisiko.

British Journal of Clinical Pharmacology Epub Jan 2010, DOl: 10.1111/j.1365-2125.2012.04124.x 